\title{
Crossing Symmetric Dispersion Relations for Mellin Amplitudes
}

\author{
Rajesh Gopakumar $\odot,{ }^{1}$ Aninda Sinha $\odot,{ }^{2}$ and Ahmadullah Zahed $\odot^{2}$ \\ ${ }^{1}$ International Centre for Theoretical Sciences (ICTS-TIFR), Shivakote, Hesaraghatta Hobli, Bangalore North 560 089, India \\ ${ }^{2}$ Centre for High Energy Physics, Indian Institute of Science, C.V. Raman Avenue, Bangalore 560012, India
}

(Received 23 February 2021; accepted 5 May 2021; published 27 May 2021)

\begin{abstract}
We consider manifestly crossing symmetric dispersion relations for Mellin amplitudes of scalar four point correlators in conformal field theories. This allows us to set up the nonperturbative Polyakov bootstrap for the conformal field theories in Mellin space on a firm foundation, thereby fixing the contact term ambiguities in the crossing symmetric blocks. Our new approach employs certain "locality" constraints replacing the requirement of crossing symmetry in the usual fixed- $t$ dispersion relation. Using these constraints, we show that the sum rules based on the two channel dispersion relations and the present dispersion relations are identical. Our framework allows us to connect with the conceptually rich picture of the Polyakov blocks being identified with Witten diagrams in anti-de Sitter space. We also give two sided bounds for Wilson coefficients for effective field theories in anti-de Sitter space.
\end{abstract}

DOI: 10.1103/PhysRevLett.126.211602

Introduction.-Conformal field theories (CFTs) are central to our modern understanding of strongly interacting systems in high energy physics, condensed matter physics, and statistical mechanics. It is therefore crucial to develop calculational tools to extract the dynamics of these theories. Four point correlation functions in CFTs are constrained by crossing symmetry and unitarity in addition to the conformal symmetries. The usual bootstrap consistency conditions impose crossing symmetry on the conformal block expansion, and this places strong constraints on the scaling dimensions of operators as well as their three point functions.

An alternative approach is to make the crossing symmetry manifest at the outset and instead impose other consistency requirements. In 1974, Polyakov [1] proposed a version of the conformal bootstrap that used crossing symmetric blocks. Consistency with the operator product expansion (OPE) led to dynamical constraints. This idea lay dormant until recently [2,3], when its power was demonstrated in Mellin space [3,4]. The latter is the natural habitat for conformal correlators, playing the role that momentum space does for flat space scattering amplitudes [5-9].

An attractive feature of this approach is that the basis of Polyakov is essentially that of exchange Witten diagrams used in the anti-de Sitter (AdS)/CFT correspondence [3,4]. Nevertheless, the nonperturbative validity of this approach

Published by the American Physical Society under the terms of the Creative Commons Attribution 4.0 International license. Further distribution of this work must maintain attribution to the author(s) and the published article's title, journal citation, and DOI. Funded by SCOAP . remained obscure. Indeed, in [10], it was pointed out that contact terms need to be added to the basis; however, it was not clear what would fix these terms. In the special case of 1D CFT, this issue was resolved in [11-13]; unfortunately, these methods do not carry over to higher dimensions. Recent works [14-17] have endeavored to clarify the nonperturbative validity of the Polyakov bootstrap in $d \geq$ 2 by considering dispersion relations in Mellin space. However, these relations exhibit only two channel symmetry, with crossing symmetry additionally imposed, thereby going against the spirit of Polyakov's original work. A nonperturbative version of the crossing symmetric bootstrap would no doubt shed light on the very successful numerical developments arising from bootstrapping position space correlators $[18,19]$. Furthermore, through the connection to Witten diagrams, such an approach is the natural one for CFTs with a large radius AdS dual.

In this Letter, we will consider a manifestly crossing symmetric dispersion relation for Mellin amplitudes, focusing on identical scalars. This builds on certain relatively obscure investigations in the 1970s [20], whose utility was recently demonstrated in the context of quantum field theories (QFTs) [21]. In this approach, we will employ a crossing symmetric parameterization of the Mellin variables $(s, t)$ (which are analogous to the usual Mandelstam invariants) and write the corresponding dispersion relation. This is to be contrasted with the more conventional fixed- $t$ dispersion relation, which has symmetry manifest only in the $(s, u)$ channels $[14,15]$.

The price we pay for making crossing symmetry manifest is the potential presence of "nonlocal" singularities. More precisely, our amplitude will generally have unphysical poles in the Mellin variables (or rather, crossing symmetric combinations thereof). Thus, in our approach, 
we need to impose "locality constraints" [Eq. (5)] for consistency. This then leads to our central claim: Once the locality constraints are imposed, the crossing symmetric dispersion relations admit a Witten diagram expansion and, moreover, all contact terms are fixed, as proposed in [10], up to a constant.

We should emphasize that the way the Witten diagrams emerge from the crossing symmetric kernel is quite nontrivial and only happens after imposition of the constraints. Compared to the approach of $[14,15]$, our locality constraints appear to be equivalent to the sum rules arising from requiring crossing symmetry (whereas their expressions do not have the unphysical poles above). This demonstrates that the expansion in Witten diagrams is robust and captures the requirements of both crossing symmetry and locality, as might be expected. In our approach and that of the fixed- $t$ dispersion relations, one then proceeds to impose the so-called Polyakov conditions. This is the requirement that there are no spurious double trace operators in the spectrum. It enables us to bootstrap the dynamical conformal data of scaling dimensions and OPE coefficients.

We note that, in the context of QFTs, the analogous locality constraints [22] were also argued [21] to be equivalent to the crossing symmetry conditions for the fixed- $t$ dispersion relation for effective field theories $[23,24]$. Analogously, we will also consider bounds on the Wilson coefficients of the Mellin amplitudes for effective scalar field theories in AdS space. We give preliminary results for two sided bounds similar to those for QFTs [21,23,24].

Crossing symmetric dispersion relation.-We define the Mellin amplitude $\mathcal{M}\left(s_{1}, s_{2}\right)$ for a four point identical scalar CFT correlator as

$$
\mathcal{G}(u, v)=\int \frac{d s_{1}}{2 \pi i} \frac{d s_{2}}{2 \pi i} u^{s_{1}+2 \Delta_{\phi} / 3} v^{s_{2}-\Delta_{\phi} / 3} \mu\left(s_{i}\right) \mathcal{M}\left(s_{1}, s_{2}\right),
$$

where $s_{3}=-s_{1}-s_{2}$, the measure factor $\mu\left(s_{i}\right)=\Gamma^{2}\left(\Delta_{\phi} / 3-s_{1}\right) \Gamma^{2}\left(\Delta_{\phi} / 3-s_{2}\right) \Gamma^{2}\left(\Delta_{\phi} / 3-s_{3}\right)$, and $\mathcal{G}(u, v)$ is the position space conformal correlator. $\Delta_{\phi}$ is the scaling dimension of the external scalars. $\mathcal{M}\left(s_{1}, s_{2}\right)$ is analogous to the usual flat space scattering amplitude, and $s_{1}, s_{2}$ are analogous to the Mandelstam invariants [related to the usual $(s, t)$ by a shift [25] ]. Motivated by $S$-matrix amplitudes, where we can write a crossing symmetric dispersion relation [20,21], we will write a similar relation for $\mathcal{M}\left(s_{1}, s_{2}\right)$, assuming its nonperturbative existence in a region of the complex plane [14]. We consider hypersurfaces $\left(s_{1}-a\right)\left(s_{2}-a\right)\left(s_{3}-a\right)=-a^{3}$, with $a$ being a real parameter. The $s_{i}$ can then be parameterized as

$$
s_{k}(z, a)=a-\frac{a\left(z-z_{k}\right)^{3}}{z^{3}-1}, \quad k=1,2,3,
$$

where $z_{k}$ are cube roots of unity. Note that $a=$ $\left[s_{1} s_{2} s_{3} /\left(s_{1} s_{2}+s_{2} s_{3}+s_{3} s_{1}\right)\right] \quad$ is crossing symmetric. $\mathcal{M}\left(s_{1}, s_{2}\right)$ is an analytic function of $(z, a)$. Unlike the $S$ matrix amplitude, CFT Mellin amplitudes have poles in the twist of the exchanged operator (with accumulation points) rather than cuts. Instead of a dispersion relation in $s$ for fixed $t$, we now write (a twice subtracted) relation in $z$ for fixed $a$, as in [20,21]. In our case, this becomes, following steps similar to $[20,21]$ (see supplementary material A of [21]),

$$
\begin{aligned}
\mathcal{M}\left(s_{1}, s_{2}\right)= & \alpha_{0}+\frac{1}{\pi} \int_{\tau^{(0)}}^{\infty} \frac{d s_{1}^{\prime}}{s_{1}^{\prime}} \mathcal{A}\left[s_{1}^{\prime} ; s_{2}^{\prime}\left(s_{1}^{\prime}, a\right)\right] \\
& \times H\left(s_{1}^{\prime} ; s_{1}, s_{2}, s_{3}\right),
\end{aligned}
$$

where $\alpha_{0}=\mathcal{M}\left(s_{1}=0, s_{2}=0\right)$ is a subtraction constant, $\mathcal{A}\left(s_{1} ; s_{2}\right)$ is the $s$-channel discontinuity, and

$$
\begin{aligned}
H\left(s ; s_{1}, s_{2}, s_{3}\right) & =\left(\frac{s_{1}}{s-s_{1}}+\frac{s_{2}}{s-s_{2}}+\frac{s_{3}}{s-s_{3}}\right) \\
s_{2}^{\prime}(s, a) & =-\frac{s}{2}\left[1-\left(\frac{s+3 a}{s-a}\right)^{1 / 2}\right],
\end{aligned}
$$

which defines the crossing symmetric kernel $H$ [20,21]. Here we have solved for $s_{2}^{\prime}$ for fixed $a$ in terms of the other independent variable $s_{1}^{\prime}$ from the defining relation below [Eq. (1)]. Note that Eq. (2) is manifestly three channel crossing symmetric since the dependence of $s_{2}^{\prime}$ is only through $a$. In the Supplemental Material [26], we have numerically investigated how well Eq. (2) represents the 2D Ising model Mellin amplitude. We have also examined there the conformal partial wave expansion from which we can work out the domain of $a$ where Eq. (2) converges. Let $\tau^{(0)}$ be the starting point of the chain of poles in $s_{1}$. Following [15], the conformal partial wave expansion converges when $-\tau^{(0)} / 3 \leq \operatorname{Re}(a)<2 \tau^{(0)} / 3$ for $\tau^{(0)}>0$ and $\tau^{(0)}<\operatorname{Re}(a)<2 \tau^{(0)} / 3$ for $\tau^{(0)}<0$.

Locality constraints. - The Mellin amplitude of identical scalars has complete crossing symmetry and hence admits a manifestly symmetric expansion,

$\mathcal{M}\left(s_{1}, s_{2}\right)=\sum_{p, q=0}^{\infty} \mathcal{M}_{p, q} x^{p} y^{q}=\sum_{p, q=0}^{\infty} \mathcal{M}_{p, q} x^{p+q} a^{q}$,

with $x=-\left(s_{1} s_{2}+s_{2} s_{3}+s_{3} s_{1}\right)$ and $y=-s_{1} s_{2} s_{3}$ (with $y=a x$ ). It is important that only positive powers of $x, y$ appear in this expansion around the crossing symmetric point $(x=y=0)$ so as not to have unphysical singularities. However, as we will see, this will not be obviously evident for our dispersion relation. Thus, we will need to impose the nontrivial constraints

$$
\mathcal{M}_{p, q}=0, \quad p<0 .
$$


(Note: $q \geq 0$ is built into our formalism.) In the fixed- $t$ dispersion relation, there are no such negative powers and the corresponding sum rules turn out to follow from requiring crossing invariance [27]. In our approach, on the other hand, the constraints in Eq. (5) are the only additional ones we need to impose, apart from the Polyakov conditions to be discussed below.

Crossing symmetric block expansion.-The s-channel discontinuity in Eq. (2) comes from the series of poles in the twist (including accumulation points):

$$
\mathcal{A}\left(s_{1}, s_{2}\right)=\pi \sum_{\Delta, \ell, k}^{\infty} c_{\Delta, \ell}^{(k)} P_{\Delta, \ell}\left(\tau_{k}, s_{2}\right) \delta\left(\tau_{k}-s_{1}\right) .
$$

Here $c_{\Delta, \ell}^{(k)}$ is proportional to the square of the OPE coefficient and is explicitly given in the Supplemental Material. The $P_{\Delta, \ell}\left(s_{1}, s_{2}\right)$ are the Mack polynomials that are the building blocks of the conformal partial wave expansion and are also given for reference in the Supplemental Material. We have also defined the twist $\tau_{k}=$ $(\Delta-\ell) / 2+k-\left(2 \Delta_{\phi} / 3\right)$ with integer $k \geq 0$. Using these, the dispersion relation [Eq. (2)] reads as [note: $\tau_{k} \geq \tau^{(0)}$ ]

$\mathcal{M}\left(s_{1}, s_{2}\right)=\alpha_{0}+\sum_{\Delta, \ell, k}^{\infty} \frac{c_{\Delta, \ell}^{(k)}}{\tau_{k}} \mathcal{Q}_{\ell, k}^{(\Delta)}(a) H\left(\tau_{k} ; s_{1}, s_{2}, s_{3}\right)$,

where $s_{3}=-s_{1}-s_{2}$ and

$$
\mathcal{Q}_{\ell, k}^{(\Delta)}(a) \equiv P_{\Delta, \ell}\left[\tau_{k}, s_{2}^{\prime}\left(\tau_{k}, a\right)\right] .
$$

The final answer [Eq. (6)] is fully crossing symmetric. Notice that since $s_{2}^{\prime}\left(\tau_{k}, a\right)_{s_{1}=\tau_{k}}=s_{2}$, Eq. (6) gives the correct residues at the poles $\tau_{k}$ in each channel. In the Supplemental Material, we perform several checks of the convergence of the representation [Eq. (6)].

Witten diagram expansion.-We can now relate the block expansion in Eq. (6) to the Witten diagram expansion. Note that the $s$-channel Witten diagram can be written in terms of the meromorphic pieces [10]:

$$
M_{\Delta, \ell, k}^{(s)}\left(s_{1}, s_{2}\right)=P_{\Delta, \ell}\left(s_{1}, s_{2}\right)\left(\frac{1}{\tau_{k}-s_{1}}-\frac{1}{\tau_{k}}\right) .
$$

Here, for convenience, we have subtracted an additional polynomial piece $\propto 1 / \tau_{k}$ compared to [10]. The $t$, $u$-channel Witten diagrams are related via $M_{\Delta, \ell, k}^{(t)}\left(s_{1}, s_{2}\right)=$ $M_{\Delta, \ell, k}^{(s)}\left(s_{2}, s_{3}\right), M_{\Delta, \ell, k}^{(u)}\left(s_{1}, s_{2}\right)=M_{\Delta, \ell, k}^{(s)}\left(s_{3}, s_{1}\right)$.

The key observation is the following. Since $\left[1 /\left(\tau_{k}-s_{1}\right)-1 / \tau_{k}\right]=\left(1 / \tau_{k}\right)\left[s_{1} /\left(\tau-s_{1}\right)\right]$, the crossing symmetric kernel $H\left(\tau_{k} ; s_{1}, s_{2}, s_{3}\right)$ in Eq. (3) also has the same meromorphic pieces, in each channel, as the Witten exchange diagrams. However, the difference between the expansion [Eq. (6)] and the expansion in terms of the individual Witten diagrams arises in the prefactor. In the
Witten diagram [Eq. (8)], the prefactor is the usual polynomial dependence on the Mellin variables in $P_{\Delta, \ell}\left(s_{1}, s_{2}\right)$, while the prefactor in Eq. (6) is the same Mack polynomial but with an argument $s_{2}^{\prime}$, as shown in Eq. (7).

Now, from Eq. (3), we see that $s_{2}^{\prime}\left(\tau_{k}, a\right)$ has singularities when $a=y / x=\tau_{k}$. These are unphysical since they individually give terms in the expansion that should not be present in the full amplitude. Indeed, as we will see explicitly below, they will give terms with negative powers of $x$ and thus an expansion that is not of the form in Eq. (4). Note that $H\left(\tau_{k} ; s_{1}, s_{2}, s_{3}\right)=\left\{\left[x\left(2 \tau_{k}-3 a\right)\right] /\left[x a-x \tau_{k}+\left(\tau_{k}\right)^{3}\right]\right\}$; therefore, we can expand Eq. (6) around $a=0, x=0$ to get only positive powers in the expansion [Eq. (4)]. Thus, what we need to do is to simultaneously expand Eq. (7) in powers of $a$ and set the net negative powers of $x$ to zero, as per Eq. (5). Since the meromorphic pieces of Eq. (6) coincide with those of the exchange Witten diagrams, the net result is to remove the spurious singularities coming from $s_{2}^{\prime}\left(\tau_{k}, a\right)$. This leaves a finite number of polynomial contact pieces, which are all now fixed up to a constant.

Let us illustrate the procedure. Consider the difference

$$
\mathcal{D}_{\ell}=\frac{1}{\tau_{k}} \mathcal{Q}_{\ell, k}^{(\Delta)}(a) H\left(\tau_{k} ; s_{1}, s_{2}, s_{3}\right)-\sum_{i=s, t, u} M_{\Delta, \ell, k}^{(i)}\left(s_{1}, s_{2}\right) .
$$

Given that the Mack polynomial is of order $\ell$ in the variables, $\mathcal{Q}_{\ell, k}^{(\Delta)}(a)$ and thus the difference $\mathcal{D}_{\ell}$ will have an unphysical pole of order $\ell / 2$ at $a=\tau_{k}$. As a concrete example, take the $\ell=2$ block. The Mack polynomial has a finite expansion $P_{\Delta, \ell}\left(s_{1}, s_{2}\right)=\sum_{m=0, n=0}^{m+n \leq \ell}\left(s_{1}\right)^{m}\left(s_{2}\right)^{n} b_{m, n}^{(\ell)}$. Thus, in our case, we have $P_{\Delta, \ell=2}\left(s_{1}, s_{2}\right)=s_{2}^{2} b_{0,2}^{(2)}+s_{1}^{2} b_{2,0}^{(2)}+s_{1} s_{2} b_{1,1}^{(2)}+$ $s_{1} b_{1,0}^{(2)}+s_{2} b_{0,1}^{(2)}+b_{0,0}^{(2)}$, and therefore we find

$$
\mathcal{D}_{\ell=2}=\frac{x b_{0,2}^{(2)}}{a-\tau_{k}}+\frac{x\left(b_{0,2}^{(2)}+2 b_{2,0}^{(2)}\right)}{\tau_{k}} .
$$

As expected, there is a single pole at $a=y / x=\tau_{k}$ that gives rise to negative powers in $x, y$, as well as additional polynomial pieces. In other words, $\mathcal{D}_{\ell=2}$ takes the form

$$
\mathcal{D}_{\ell=2}=\frac{2 x b_{2,0}^{(2)}}{\tau_{k}}-\frac{y b_{0,2}^{(2)}}{\tau_{k}^{2}}-\left[x b_{0,2}^{(2)}\right] \sum_{n=2}^{\infty} a^{n} \tau_{k}^{-n-1} .
$$

The last term will not contribute once the requirement of Eq. (5) is imposed (on the full amplitude). The first two terms are polynomials as expected and given by

$$
M_{\Delta, \ell=2, k}^{(c)}\left(s_{1}, s_{2}\right)=\frac{2 x b_{2,0}^{(2)}}{\tau_{k}}-\frac{y b_{0,2}^{(2)}}{\tau_{k}^{2}} .
$$

It should be clear that similar arguments hold for all $\ell$ - the corresponding $\mathcal{D}_{\ell}$ will have spurious singularities that 
when expanded in powers of $a$ give negative powers of $x$ except for a finite number of pieces that are polynomials in $x$, $y$. Once the locality constraints are imposed on the full amplitude, only these polynomial pieces survive. The $\ell=4$ case is also discussed explicitly in the Supplemental Material.

We have therefore argued for the Witten diagram expansion of the Mellin amplitude as

$\mathcal{M}\left(s_{1}, s_{2}\right)=\sum_{\Delta, \ell, k}^{\infty} c_{\Delta, \ell}^{(k)}\left[M_{\Delta, \ell, k}^{(c)}\left(s_{1}, s_{2}\right)+\sum_{i=s, t, u} M_{\Delta, \ell, k}^{(i)}\left(s_{1}, s_{2}\right)\right]$.

$M_{\Delta, \ell, k}^{(c)}\left(s_{1}, s_{2}\right)$ are the polynomial pieces (for each $\ell$ ) in $\mathcal{D}_{\ell}$ after removing the spurious singularities at $a=\tau_{k}$. These are precisely the contact terms that were not fixed in [10]. Note that for the $\ell=0$ case, $M_{\Delta, \ell=0, k}^{(c)}\left(s_{1}, s_{2}\right)$ is a constant that gives the subtraction constant $\alpha_{0}$. In $d=1$, where there are no spins, this is the only contact term, as discussed in detail in [13]. Our conclusion, then, is that the contact term ambiguity anticipated in [10] is fixed for any spin, and the Witten diagram expansion holds once the locality constraints are satisfied. Furthermore, the explicit form of the contact term shows why it did not contribute to the $O\left(\epsilon^{3}\right)$ results in $[3,10]$, i.e., it can been seen to start contributing only at $O\left(\epsilon^{4}\right)$.

Polyakov conditions and sum rules. - The measure factor $\mu\left(s_{i}\right)$ in the definition of the Mellin amplitude introduces additional spurious poles in the full amplitude. We must therefore impose the condition that this expansion not have the poles for double trace operators with $\Delta=2 \Delta_{\phi}+\ell+2 p$. Thus, $\mathcal{M}\left(s_{1}, s_{2}\right)$ must vanish at $s_{1}=\Delta_{\phi} / 3+p \quad[3,10,14]$, which are the so-called Polyakov conditions. From Eq. (6), we get

$$
\mathfrak{\mho}_{p}\left(s_{2}\right) \equiv \mathcal{M}\left(s_{1}=\frac{\Delta_{\phi}}{3}+p, s_{2}\right)=0 .
$$

Derivation of sum rules in [15].-We define the combinations for $p_{i} \in \mathbb{Z}^{\geq 0}$

$$
\begin{aligned}
\Omega_{p_{1}, p_{2}, p_{3}}\left(s_{2}\right) \equiv & -\frac{\mathfrak{F}_{p_{1}}\left(s_{2}\right)}{\left(p_{1}-p_{2}\right)\left(p_{1}+p_{3}+s_{2}+\frac{2 \Delta_{\phi}}{3}\right)} \\
& -\frac{\mathfrak{F}_{p_{2}}\left(s_{2}\right)}{\left(p_{2}-p_{1}\right)\left(p_{2}+p_{3}+s_{2}+\frac{2 \Delta_{\phi}}{3}\right)} \\
& -\frac{\mathfrak{F}_{p_{3}}\left(s_{2}\right)}{\left(p_{1}+p_{3}+s_{2}+\frac{2 \Delta_{\phi}}{3}\right)\left(p_{2}+p_{3}+s_{2}+\frac{2 \Delta_{\phi}}{3}\right)},
\end{aligned}
$$

with $\mathfrak{\mho}_{p}$ defined in (13). We can now compare $\Omega_{p_{1}, p_{2}, p_{3}}\left(s_{2}\right)$ to the functional $\omega_{p_{1}, p_{2}, p_{3}}$ in [15]. In our notation,

$$
\begin{aligned}
\omega_{p_{1}, p_{2}, p_{3}}\left(s_{2}\right)= & \sum_{\Delta, \ell, k}^{\infty} c_{\Delta, \ell}^{(k)} P_{\Delta, \ell}\left(\tau_{k}, s_{2}\right) \\
& \times\left[\frac{1}{\prod_{i=1}^{2}\left(\frac{\Delta_{\phi}}{3}+p_{i}-\tau_{k}\right)\left(\frac{\Delta_{\phi}}{3}+p_{3}+\tau_{k}+s_{2}\right)}\right. \\
& \left.-\frac{1}{\prod_{i=1}^{2}\left(\frac{\Delta_{\phi}}{3}+p_{i}+s_{2}+\tau_{k}\right)\left(\frac{\Delta_{\phi}}{3}+p_{3}-\tau_{k}\right)}\right] .
\end{aligned}
$$

If we now Taylor expand $\omega_{p_{1}, p_{2}, p_{3}}\left(s_{2}\right)$ and $\Omega_{p_{1}, p_{2}, p_{3}}\left(s_{2}\right)$ around $s_{2}=0$, we get, respectively,

$$
\begin{aligned}
& \Omega_{p_{1}, p_{2}, p_{3}}\left(s_{2}\right)=\sum_{r=0}^{\infty}\left(s_{2}\right)^{r} \Omega_{p_{1}, p_{2}, p_{3}}^{(r)} \\
& \omega_{p_{1}, p_{2}, p_{3}}\left(s_{2}\right)=\sum_{r=0}^{\infty}\left(s_{2}\right)^{r} \omega_{p_{1}, p_{2}, p_{3}}^{(r)}
\end{aligned}
$$

and find that $\omega_{p_{1}, p_{2}, p_{3}}^{(i)}=\Omega_{p_{1}, p_{2}, p_{3}}^{(i)}$ for $i=1, \ldots, 5$. From $i=$ 6 onward, there are nontrivial relations. To give a flavor of these relations, we exhibit, for $r=6$ and $r=7$ (where we have put some of the $p_{i}$ to zero),

$$
\omega_{p_{1}, p_{2}, 0}^{(6)}-\Omega_{p_{1}, p_{2}, 0}^{(6)} \propto \mathcal{M}_{-1,2}
$$

and

$\omega_{p_{1}, 0,0}^{(7)}-\Omega_{p_{1}, 0,0}^{(7)} \propto \mathcal{M}_{-1,2}-\frac{\Delta_{\phi}\left(\Delta_{\phi}+3 p_{1}\right)}{2 \Delta_{\phi}+3 p_{1}} \mathcal{M}_{-2,3}$.

The explicit expressions for the $\mathcal{M}_{p, q}$ appearing here are given below in Eq. (19). What these relations indicate is that once the locality constraints [Eq. (5)] are imposed, our Polyakov conditions are the same as those in [15]. We have checked that similar relations hold for any $\left(p_{1}, p_{2}, p_{3}\right)$ for higher $r$.

Positivity conditions for CFTs. - Positivity conditions on amplitudes are important for putting bounds on the Wilson coefficients of effective field theories (EFTs) in a dual AdS spacetime. We obtain them in our formalism following [21]. From Eq. (6) (see Supplemental Material), we find

$$
\mathcal{M}_{n-m, m}=\sum_{\Delta, \ell, k}^{\infty} c_{\Delta, \ell}^{(k)} \mathcal{B}_{n, m}^{(\Delta, \ell, k)}, \quad n \geq 1
$$

Here $\mathcal{B}_{n, m}^{(\Delta, \ell, k)}$ is given by

$$
\mathcal{B}_{n, m}^{(\Delta, \ell, k)}=\sum_{q=0}^{m} \mathfrak{U}_{n, m, q}^{\left(\tau_{k}\right)}(-1)^{m+q} P_{\Delta, \ell ; q}\left(\tau_{k}, 0\right),
$$

where $P_{\Delta, \ell ; q}\left(\tau_{k}, 0\right)=\left.\partial_{s_{2}}^{q} P_{\Delta, \ell}\left(\tau_{k}, s_{2}\right)\right|_{s_{2}=0}$ and 


$$
\begin{aligned}
\mathfrak{U}_{n, m, q}^{\left(\tau_{k}\right)}= & \frac{(n-q-1) !(m+2 n-3 q)}{q !(n-m) !(m-q) !\left(\tau_{k}\right)^{m+2 n-q+1}} \\
& \times{ }_{4} F_{3}\left[\begin{array}{c}
\frac{q}{2}+\frac{1}{2}, \frac{q}{2}, q-m, q+1-\frac{2 n+m}{3} \\
q+1, q+1-n, q-\frac{2 n+m}{3}
\end{array} ; 4\right] .
\end{aligned}
$$

One can check numerically that (for $\tau_{k=0} \geq 0$ ) $P_{\Delta, \ell ; q}\left(\tau_{k}, 0\right) \geq 0$. A similar claim was made in [14]. Also we find that $\mathfrak{U}_{n, m, q}^{\left(\tau_{k}\right)} \geq 0$. As in [21], we can search for $\chi_{n}^{(r, m)}\left(\tau_{k}\right)$ such that

$$
\sum_{r=0}^{m} \chi_{n}^{(r, m)}\left(\tau_{k}\right) \mathcal{B}_{n, r}^{(\Delta, \ell, k)}=\mathfrak{U}_{n, m, m}^{\left(\tau_{k}\right)}(-1)^{m+q} P_{\Delta, \ell ; q}\left(\tau_{k}, 0\right) \geq 0 .
$$

Next, define $\mathcal{M}^{(0)}\left(s_{1}, s_{2}\right)$, the amplitude, after subtracting off the twist zero contributions (if any). Let $\tau^{(0)}$ denote the minimum nonzero twist $\tau_{k=0}$. Using this in Eq. (19), we can write down positivity constraints on $\mathcal{M}_{n-m, m}^{(0)}$ similar to those in [21] [with $\tau^{(0)} \geq 0$ ]:

$$
\mathcal{M}_{n-m, m}^{(0)}+\sum_{r=0}^{m-1} \chi_{n}^{(r, m)}\left[\tau^{(0)}\right] \mathcal{M}_{n-r, r}^{(0)} \geq 0
$$

A recursion relation for $\chi_{n}$ was worked out in [21]. For example [28], $\quad \chi_{n}^{(0,1)}\left(\tau_{k}\right)=(2 n+1) / 2 \tau_{k}, \quad \chi_{n}^{(0,2)}\left(\tau_{k}\right)=$ $[2 n(n+2)+3] / 4\left(\tau_{k}\right)^{2}, \chi_{n}^{(1,2)}\left(\tau_{k}\right)=(2 n+1) / 2 \tau_{k}$. These imply, for instance, $\mathcal{M}_{0,1}^{(0)}>-\left[3 / 2 \tau^{(0)}\right] \mathcal{M}_{1,0}^{(0)}$.

Two-sided bounds.-If we assume that higher spin partial waves are suppressed, then we can argue for the existence of two-sided bounds. We can check numerically that for $\ell<\ell_{*}$, there always exists some positive $\beta=O\left(\ell_{*}\right)$ such that

$$
\mathcal{B}_{1,1}^{(\Delta, \ell, k)}<\beta \mathcal{B}_{1,0}^{(\Delta, \ell, k)}, \quad \forall \Delta_{\phi} \in\left(\frac{\alpha}{2}, \frac{3 \alpha}{4}\right),
$$

where $\alpha=d / 2-1$. Therefore, we get a two-sided bound valid for $\alpha<2 \Delta_{\phi}<\frac{3}{2} \alpha$ :

$$
-\frac{3}{2 \tau^{(0)}} \mathcal{M}_{1,0}^{(0)}<\mathcal{M}_{0,1}^{(0)}<\beta \mathcal{M}_{1,0}^{(0)}
$$

To derive a stronger upper bound analogous to the QFT bounds in $[23,24]$, we will need to incorporate the locality constraints as well, a detailed study of which we leave for future work. In the case of AdS EFT for a scalar with mass $m$, with the AdS radius given by $R$, we show in the Supplemental Material that, for $m R \gg 1$,

$$
\mathcal{M}_{0,1}^{(\mathrm{AdS})}<\left[1+\frac{\alpha}{2(2 \alpha+3) m^{2} R^{2}}\right] \frac{(10 \alpha+11)}{(2 \alpha+1) \delta_{0}} \mathcal{M}_{1,0}^{(\mathrm{AdS})},
$$

where $\delta_{0}$ is related to the EFT scale. Besides the fact that a two-sided bound in AdS space is novel, the derivation using our approach is algebraically simpler compared to the fixed- $t$ dispersion. In the future, it will be desirable to understand the properties of $\mathcal{B}_{m, n}^{(\Delta, \ell, k)}$ better.

Discussion.-The new framework we have developed in this Letter has allowed us to make contact with Polyakov's original crossing symmetric bootstrap proposal and has shown the equivalence, in a nontrivial way, with the results of [14]. Furthermore, this approach clarifies the underlying reason for the existence of Witten diagram representations for general CFT correlators. To this end, it will be good to broaden the preliminary discussion in the Supplemental Material on the convergence of the Witten diagram expansion and apply it to new settings. It will also be useful to compare the results developed in this Letter to the position space results in, e.g., [29,30]. Mellin space expressions, in turn, permit ready comparison with flat space results. Many of the positivity conditions for (combinations of) Gegenbauer polynomials appear to extend to Mack polynomials. Mathematically, it will be useful to develop proofs of these relations, which will enable a systematic study of corrections to EFTs in AdS space in the large radius limit. Finally, we hope that this work will give new impetus to numerical bootstrap studies using the constraints and functionals [such as in Eq. (14)] defined here.

We thank Parijat Dey, Kausik Ghosh, Apratim Kaviraj, and Miguel Paulos for useful discussions. R. G.'s research is supported by a J. C. Bose fellowship of the DST as well as project RTI4001 of the Department of Atomic Energy, Government of India.

[1] A. M. Polyakov, Nonhamiltonian approach to conformal quantum field theory, Zh. Eksp. Teor. Fiz. 66, 23 (1974).

[2] K. Sen and A. Sinha, On critical exponents without Feynman diagrams, J. Phys. A 49, 445401 (2016).

[3] R. Gopakumar, A. Kaviraj, K. Sen, and A. Sinha, Conformal Bootstrap in Mellin Space, Phys. Rev. Lett. 118, 081601 (2017).R. Gopakumar, A. Kaviraj, K. Sen, and A. Sinha, A Mellin space approach to the conformal bootstrap, J. High Energy Phys. 05 (2017) 027.

[4] P. Dey, A. Kaviraj, and A. Sinha, Mellin space bootstrap for global symmetry, J. High Energy Phys. 07 (2017) 019; P. Dey, K. Ghosh, and A. Sinha, Simplifying large spin bootstrap in Mellin space, J. High Energy Phys. 01 (2018) 152.

[5] G. Mack, D-independent representation of conformal field theories in $\mathrm{D}$ dimensions via transformation to auxiliary dual resonance models. Scalar amplitudes, arXiv:0907.2407.

[6] J. Penedones, Writing CFT correlation functions as AdS scattering amplitudes, J. High Energy Phys. 03 (2011) 025.

[7] M. F. Paulos, Towards Feynman rules for Mellin amplitudes, J. High Energy Phys. 10 (2011) 074. 
[8] L. Rastelli and X. Zhou, Mellin Amplitudes for $\mathrm{AdS}_{5} \times S^{5}$, Phys. Rev. Lett. 118, 091602 (2017).

[9] L.F. Alday, On Genus-one String Amplitudes on $\mathrm{AdS}_{5} \times S^{5}$, J. High Energy Phys. 04 (2021) 005 .

[10] R. Gopakumar and A. Sinha, On the Polyakov-Mellin bootstrap, J. High Energy Phys. 12 (2018) 040.

[11] D. Mazac and M. F. Paulos, The analytic functional bootstrap. Part II. Natural bases for the crossing equation, J. High Energy Phys. 02 (2019) 163.

[12] D. Mazáč, A crossing-symmetric OPE inversion formula, J. High Energy Phys. 06 (2019) 082.

[13] P. Ferrero, K. Ghosh, A. Sinha, and A. Zahed, Crossing symmetry, transcendentality and the Regge behaviour of $1 \mathrm{~d}$ CFTs, J. High Energy Phys. 07 (2020) 170.

[14] J. Penedones, J. A. Silva, and A. Zhiboedov, Nonperturbative Mellin amplitudes: Existence, properties, applications, J. High Energy Phys. 08 (2020) 031.

[15] D. Carmi, J. Penedones, J. A. Silva, and A. Zhiboedov, Applications of dispersive sum rules: $\epsilon$-expansion and holography, arXiv:2009.13506.

[16] S. Caron-Huot, D. Mazac, L. Rastelli, and D. SimmonsDuffin, Dispersive CFT sum rules, arXiv:2008.04931.

[17] C. Sleight and M. Taronna, The unique Polyakov blocks, J. High Energy Phys. 11 (2020) 075.

[18] R. Rattazzi, V.S. Rychkov, E. Tonni, and A. Vichi, Bounding scalar operator dimensions in 4D CFT, J. High Energy Phys. 12 (2008) 031.

[19] D. Poland, S. Rychkov, and A. Vichi, The conformal bootstrap: Theory, numerical techniques, and applications, Rev. Mod. Phys. 91, 015002 (2019).

[20] G. Auberson and N. N. Khuri, Rigorous parametric dispersion representation with three-channel symmetry, Phys. Rev. D 6, 2953 (1972).
[21] A. Sinha and A. Zahed, Crossing Symmetric Dispersion Relations in QFTs, Phys. Rev. Lett. 126, 181601 (2021).

[22] In [23], these constraints were termed as "null constraints."

[23] S. Caron-Huot and V. Van Duong, Extremal effective field theories, arXiv:2011.02957.

[24] A. J. Tolley, Z. Y. Wang, and S. Y. Zhou, New positivity bounds from full crossing symmetry, arXiv:2011.02400.

[25] The notation in [10] was in terms of $s, t, u$, which are related to our $s_{i}$ via $s_{1}=s-2 \Delta_{\phi} / 3, \quad s_{2}=t-2 \Delta_{\phi} / 3$, $s_{3}=u-2 \Delta_{\phi} / 3$.

[26] See Supplemental Material at http://link.aps.org/supplemental/ 10.1103/PhysRevLett.126.211602 for additional mathematical details.

[27] For example, Eq. (141) in [14] is proportional $\mathcal{M}_{-1,2}$. In [16], these are called the "odd-spin" constraints. In fact, in the QFT context, the analogous conditions were termed as "null constraints" in [23]. In [21], it was shown that these are, again, precisely what we term locality constraints. Our formalism allows us to write these constraints in complete generality.

[28] As in [21], we find a recursion relation:

$$
\chi_{n}^{(r, m)}\left(\tau_{k}\right)=\sum_{j=r+1}^{m}(-1)^{j+r+1} \chi_{n}^{(j, m)}\left(\tau_{k}\right) \frac{\mathfrak{U}_{n, j, r}^{\left(\tau_{k}\right)}}{\mathfrak{U}_{n, r, r}^{\left(\tau_{k}\right)}}
$$

[29] A. Bissi, P. Dey, and T. Hansen, Dispersion relation for CFT four-point functions, J. High Energy Phys. 04 (2020) 092.

[30] M. F. Paulos, Dispersion relations and exact bounds on CFT correlators, arXiv:2012.10454. 